\title{
Cardiac Immobilization in Beating Heart Surgery Using Pericardial Bands
}

\author{
Srikrishna Sirivella, Isaac Gielchinsky* \\ Department of Cardiovascular and Thoracic Surgery, Newark Beth Israel Medical Center, University of Medicine \& \\ Dentistry of New Jersey, Newark, USA \\ Email: ${ }^{*}$ Igielchinsky@optonline.net
}

Received April 5, 2012; revised May 15, 2012; accepted May 28, 2012

\begin{abstract}
A novel technique of stabilization of the coronary targets with autogenous pericardial bands is described in off pump coronary revascularizations. Pericardial bands are placed across the coronary targets and snared to the pericardial well for local cardiac immobilization. This technique can be used as an alternate to mechanical stabilizers for immobilization of the coronary targets.
\end{abstract}

Keywords: Coonary Bypass Surgery; Cardiac; Off Pump CABG

\section{Introduction}

The Beating heart coronary revascularization avoids the potential harmful effects of cardiopulmonary bypass (CPB) and attendant hospital costs. The recent generation of mechanical stabilizers used for local cardiac immobilization is cumbersome to use and increases operative costs. We describe a simple technique of local cardiac immobilization by use of autogenous pericardial bands.

\section{Technique}

Following a standard median sternotomy, the surface of the anterior pericardium is freed from adipose and loose areolar tissue, from thymus down to the diaphragm, and laterally from right to left pericardio-phrenic vessels. Three pericardial bands are fashioned from the anterior pericardium extending from one pericardiophrenic vessel to another pericardiophrenic vessel as described below. Below the level of thymus, where the wide body of the pericardium begins, pericardial band 1 is raised by making two horizontal incisions $2.5 \mathrm{~cm}$ apart, extending from right pericardio-phrenic vessels to left pericardio-phrenic vessels. The length of the band is increased by connecting above two incisions inferiorly by a $2 \mathrm{~cm}$ long box incision placed parallel to left pericardio-phrenic vessels (Figure 1(A)). Pericardial band 2 is fashioned from the lower body of the pericardium by another set of two horizontal incisions $2.5 \mathrm{~cm}$ apart, extending from left pericardio-phrenic vessels to the right pericardio-phrenic vessels. This lower incision should be placed at least $3 \mathrm{~cm}$

"Corresponding author. above the pericardio-diaphragmatic border. The length of the band is increased by connecting the two incisions inferiorly by a $2 \mathrm{~cm}$ long box type incision placed parallel to the right pericardio-phrenic vessels as shown (Figure 1(A)). The remnant anterior pericardium in the midportion is incised vertically, parallel to left phrenic vessels (Figure 1(A)) to raise the pericardial band 3. All 3 pericardial bands can be fashioned within 10 minutes after a standard sternotomy incison, with each band attached to the body of the pericadium with an intact blood supply, a free body and a distal end and each measures about $2.5 \mathrm{~cm}$ wide and $10 \mathrm{~cm}$ long (Figure 1(B)).

The rest of the pericardial sac is incised and margins are sutured to the skin. Four traction sutures are placed in the pericardial well. One at the aorto-pericardial reflection, one each, $2 \mathrm{~cm}$ above the left superior and inferior pulmonary veins, and one between left pulmonary veins and inferior vena cava. Alternately a single heavy silk suture is placed in the oblique sinus incorporating a 15 $\mathrm{cm}$ vaginal tape. Traction on the above sutures or vaginal tape along with a lap pad positioned either posterior laterally to the left or inferiorly aids in a proper positioning of the heart for performing multi vessel coronary anastomoses [1].

The heart is poistioned for exposure of a selected cornoary target and the vessel is marked for the distal anastomosis. Stabilization of the coronary targets is done as follows. Two pericardial bands are placed across the target site, one proximal and another distal, exposing $1.5 \mathrm{~cm}$ length of the target vessel. The choice of the pericardial band is determined by the position of the heart and target site to be stabilized (Figures 2 and 3 ). The surgeon 
places the selected pericardial band across the target site and holds it snugged towards the pericardial well, grasping the distal portion of the band with a forceps. The operator then selects the locations on the pericardial well and on the band for placing the snares such that the suture points on the pericardial band and the well are not approximated but separated at least $1.5 \mathrm{~cm}$ distance or more. This allows for tightening of the band and stabilization of the target site if the band is snared to the pericardial well. The suture in the pericardial well should be placed at least $2 \mathrm{~cm}$ wide and $2 \mathrm{~cm}$ or more deeper to the pericardial margin and to prevent tearing of the pericardial sac when the band is snared in oppostion to the pericardial well (Figure 1(C)).

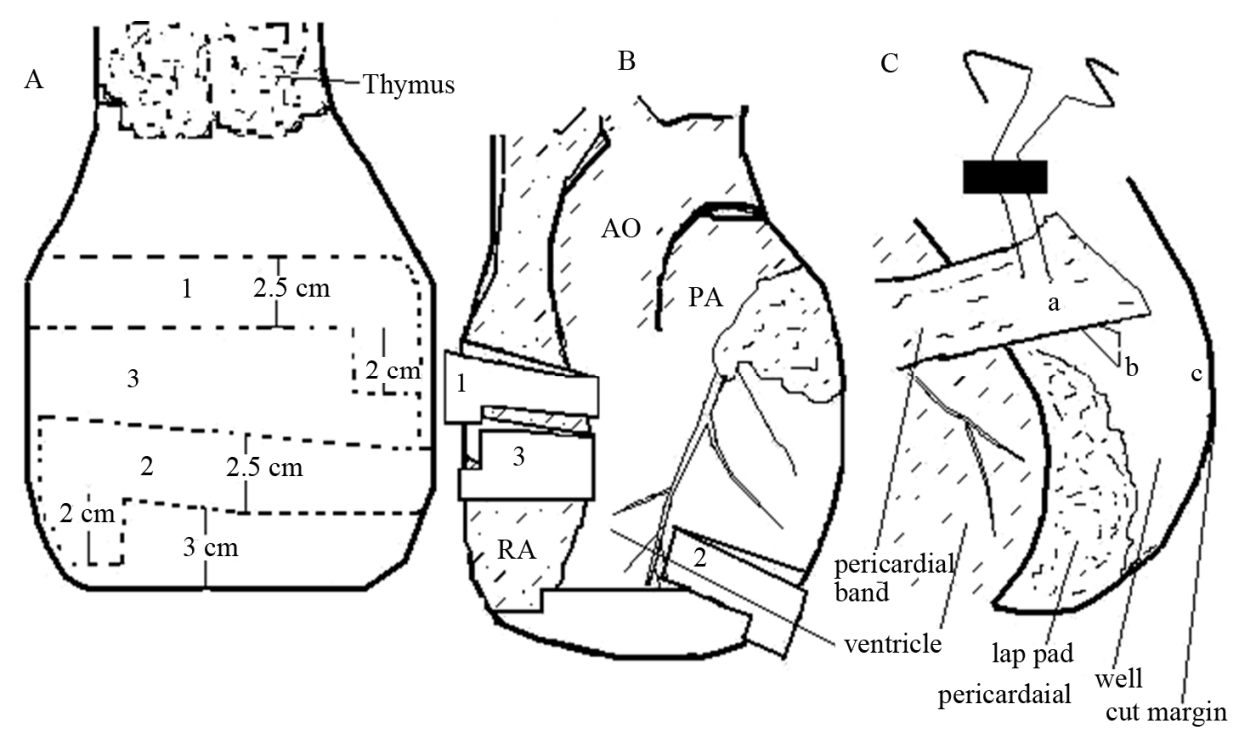

Figure 1. (A) Dotted lines show two sets of two horizontal incisions in the body of pericardium extending from one pericardio-phrenic vessel to another to raise pericardial bands 1, 2. The lower incision in each set ends $2 \mathrm{~cm}$ short of upper incision and both the incisions are connected by vertical box incision to increase the band length. Pericardial band 3 is fashioned by incising the remnant pericardium; (B) Harvested pericardial bands 1, 2, and 3 are folded backwards partially to expose the underlying heart and great vessels; (C) 2-0 prolene suture is passed through the pericardial well and selected location on the distal segment of a pericardial band. Note the suture points on the band and the well are separated by a distance (a-b) of 1.5 $\mathrm{cm}$ b-c = distance from suture location in the pericadial well to cut margin $(2 \mathrm{~cm})$. AO = ascending aorta; $P A=$ pulmonary artery; $\mathbf{R A}=$ right atrium.
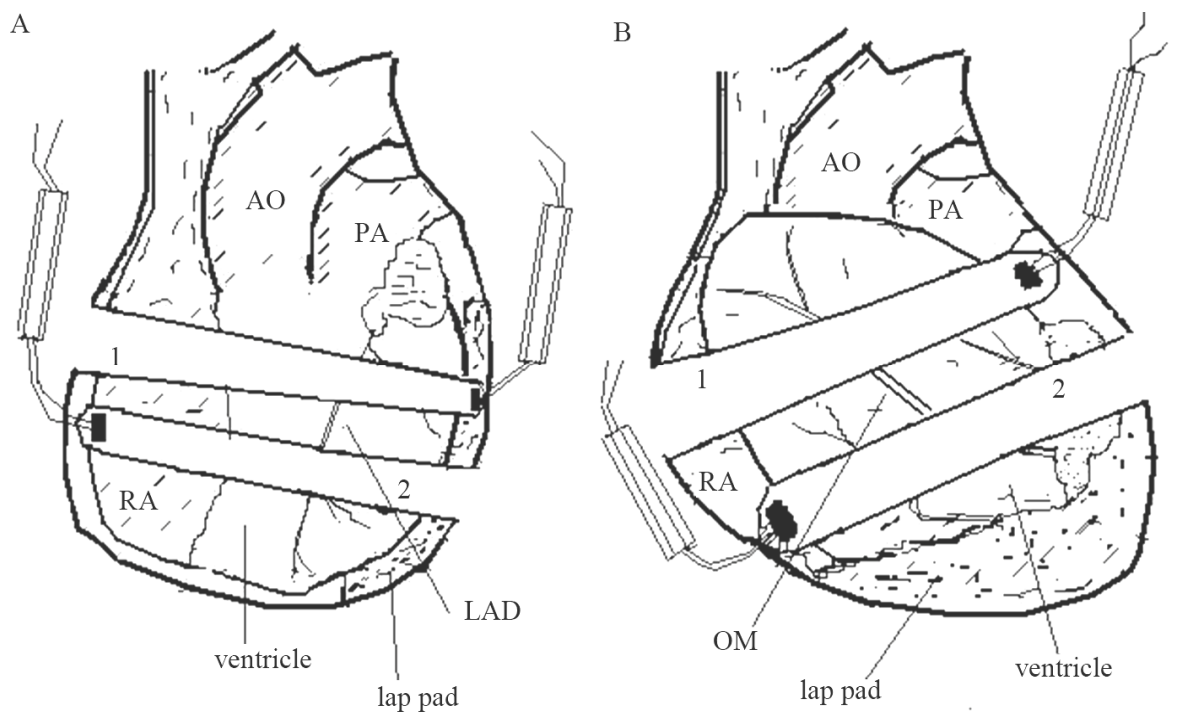

Figure 2. (A) Stabilization of LAD target by snaring pericardial bands 1 and 2 to the pericardial well. Diagonal, median and high marginal branch of circumflex coronary targets can be stabilized with same bands; (B) Stabilization of OM branch and posterolateral branch targets by snaring pericardial bands 1 and 2 to the pericardial well. Alternately bands 3 and 2 may be used. LAD = left anterior descending; OM = obtuse marginal branch of circumflex. 

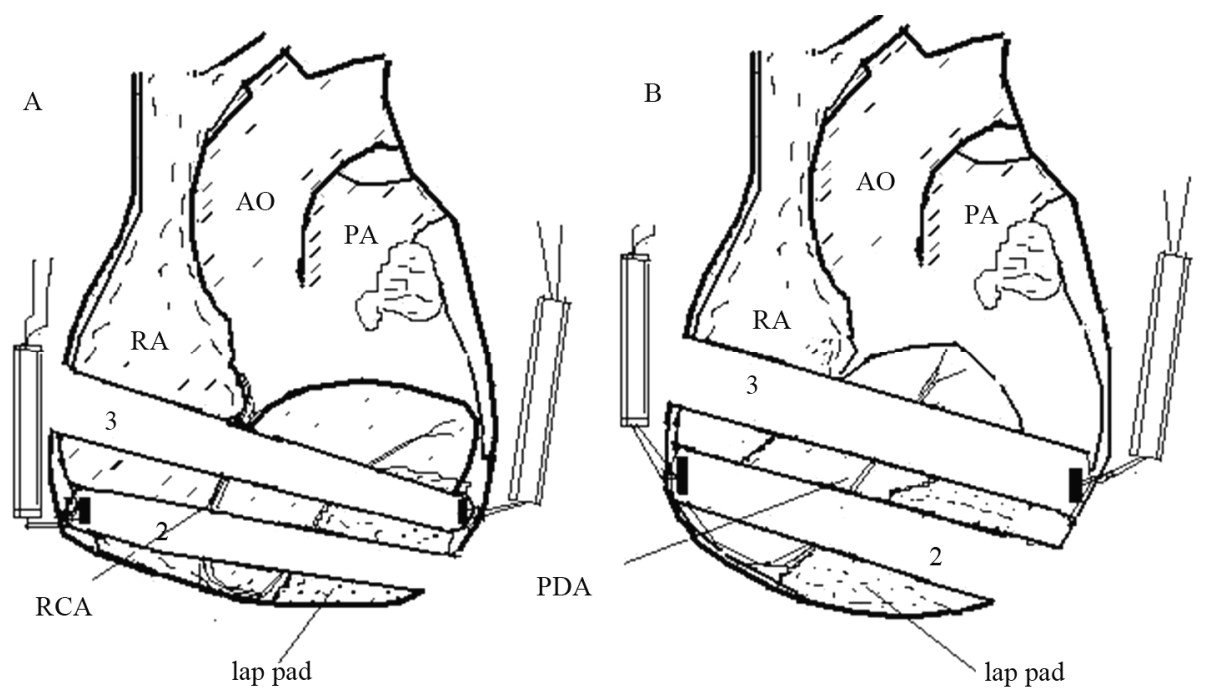

Figure 3. (A) Stabilization of distal right coronary artery target in the atrioventricular groove by snaring pericardial bands 3 and 2 to the pericardial well; (B) Stabilization of PDA target by snaring pericardial bands 3 and 2 to the pericardial well. RCA = right coronary artery; PDA = posterior descending artery.

A double arm snaring suture of 2-0 prolene is first passed through the selected locations on the pericardial well, the distal segment of the pericardial band, and then through a Teflon pledget and snared through a rubber tube (Figure 1(C)). The pericardial bands are tightened by snaring the pericardial bands to the pericardial well to stabilize the myocardium of the target site. A folded $2 \mathrm{~cm}$ wide Teflon pledget is often placed beneath the pericardial band at the target site to produce local immobilization without compressing the surrounding myocardium. The distal coronary anastomoses are performed as usual for off pump coronary revascularization procedures. A longitudinal arteriotomy is made and an anastomosis with a chosen conduit is performed using a running 7-0 prolene suture. If needed, the proximal and distal control of coronary artery is done by a loop of 5-0 prolene suture or by a silicon elastomere tape. After completion of the proximal and distal anatomoses and reversal of anticoagulation, the pericardial bands are repositioned and approximated with interrupted silk sutures to cover the heart.

\section{Comment}

Since Kolessov described beating heart coronary bypass surgery in 1964 through a left thoracotomy, a resurgence in off pump coronary revascularization has led to improvements in the technique for exposure in multivessel coronary bypass by introduction of pericardial sutures, slings, Janke net, oblique sinus sponge and vaginal tape $[2,3]$. Though earlier attempts of coronary artery surgery on a beating heart were abandoned in a favor of CPB due the lack of adequate cardiac immobilization, the introduction of mechanical stabilizers such as CTS pressure mechanical stabilizers, epicardial fixation by suction devices and the Medtronic-Utrecht Octopus system, (Med- tronic Inc., Minneapolis, MN), produced adequate cardiac immobilization of the coronary target site for performance of a safe anastomosis [3].

These devices impose additional operative costs and are cumbersome to use. The pericardial band technique is simple and adequate stabilization of multiple coronary target sites can be achieved for performance of a safe anastomosis without a crowded operative filed. The pericardial covering is restored after completion of the operative procedure and use of this technique incurs no additional cost to the patient. The disadvantages of this technique are that it can not be performed in redo surgery with pericardial adhesions and a potential for compression of myocardial territory other than the target site producing hemodynamic instability. The latter can be easily avoided by volume loading of the ventricle, proper positioning of the heart to minimize myocardial compression and by insertion of a folded Teflon felt at the target site beneath the pericardial band.

\section{REFERENCES}

[1] J. Bergsland, H. L. Karamanoukian, P. R. Soltoski and T. A. Salerno, "'Single Suture' for Circumflex Exposure in Off-Pump Coronary Artery Bypass Grafting,” The Annals of Thoracic Surgery, Vol. 68, No. 4, 1999, pp. 1428-1430. doi:10.1016/S0003 -4975(99)00935-2

[2] V. I. Kolessov, "Mammary Artery-Coronary Artery Anastomosis as Method of Treatment for Angina Pectoris," Journal of Thoracic and Cardiovascular Surgery, Vol. 54, No. 4, 1967, pp. 535-544

[3] T. H. Spooner, J. C. Hart and J. Pym, "A Two-Year, Three Institution Experience with the Medtronic Octopus: Systematic Off-Pump Surgery,” The Annals of Thoracic Surgery, Vol. 68, No. 4, 1999, pp. 1478-1481. doi:10.1016/S0003-4975(99)00966-2 